\title{
Gastrodin inhibits high glucose-induced inflammation, oxidative stress and apoptosis in podocytes by activating the AMPK/Nrf2 signaling pathway
}

\author{
LUYAN HUANG $^{1}$, MINGHAI SHAO ${ }^{2}$ and YAN ZHU ${ }^{3}$ \\ ${ }^{1}$ Department of Traditional Chinese Medicine, Zhongshan Hospital (Minhang Branch), Fudan University, \\ Shanghai 201199; ${ }^{2}$ Department of Nephrology, Shuguang Hospital Affiliated to Shanghai University of \\ Traditional Chinese Medicine, Shanghai 200021; ${ }^{3}$ Department of Traditional Chinese Medicine, \\ Shanghai Fourth People's Hospital Affiliated to Tongji University School of Medicine, Shanghai 200434, P.R. China
}

Received October 27, 2020; Accepted June 14, 2021

DOI: $10.3892 /$ etm.2021.11091

\begin{abstract}
Diabetic nephropathy (DN) is a serious and common complication of type 1 and 2 diabetes. Gastrodin has been reported to suppress high glucose (HG)-induced inflammation and oxidative stress in vivo and in vitro. However, the effect of gastrodin on DN has not been fully elucidated. The present study aimed to investigate the underlying mechanism involved in the effect of gastrodin on podocyte injury caused by DN. Cell viability was evaluated using Cell Counting Kit- 8 assay and secretion levels of TNF- $\alpha$, IL- $1 \beta$ and IL- 6 were measured using ELISA. The levels of malondialdehyde, activities of lactate dehydrogenase and superoxide dismutase were quantified using corresponding assay kits. Additionally, cell apoptosis was analyzed by TUNEL assay, whilst protein expressions related to inflammation, apoptosis and the 5'-AMP-activated protein kinase (AMPK)/nuclear factor erythroid 2-related factor 2 (Nrf2) signaling pathway were measured by western blot analysis. The results showed that gastrodin increased the viability of MPC5 cells following HG stimulation. Gastrodin also alleviated HG-induced inflammation, oxidative stress and apoptosis in MPC5 cells. Furthermore, gastrodin promoted activation of the AMPK/Nrf2 pathway in MPC5 cells. Treatment with the AMPK inhibitor, compound C, reversed the inhibitory effects of gastrodin on inflammation, oxidative stress and cell apoptosis. To conclude, treatment of MPC5 cells with gastrodin can attenuate HG-induced inflammation, oxidative stress and cell apoptosis by activating the AMPK/Nrf2
\end{abstract}

Correspondence to: Professor Yan Zhu, Department of Traditional Chinese Medicine, Shanghai Fourth People's Hospital Affiliated to Tongji University School of Medicine, 1279 Sanmen Road, Shanghai 200434, P.R. China

E-mail: yz_lh2004@126.com

Key words: gastrodin, diabetic nephropathy, podocytes, apoptosis, 5'AMP-activated protein kinase, nuclear factor erythroid 2-related factor 2 signaling pathway signaling pathway. Results from the current study suggest that gastrodin can be used as an effective therapeutic agent against HG-induced podocyte injury in DN.

\section{Introduction}

Diabetic nephropathy (DN) is a serious and common complication of type 1 and 2 diabetes (1-3). DN can eventually lead to end-stage renal disease in $\leq 40 \%$ patients with diabetes (1-3). It has been previously reported that $\mathrm{DN}$ can be considered as the strongest predictor of mortality $(4,5)$. Advanced DN is characterized by glomerular sclerosis, tubulointerstitial degeneration and fibrosis, which may result in a precipitous decline in the glomerular filtration rate, resulting in proteinuria (6-8). Previous studies have shown that podocyte damage can trigger a number of pathological changes, including glomerular sclerosis and renal failure $(9,10)$. Furthermore, it has been reported that podocyte damage is closely associated with the pathogenesis of several kidney-related diseases, including focal segmental glomerulosclerosis, minimal change nephropathy and DN $(11,12)$. Therefore, preventing podocyte injury should be considered to be an effective strategy for treating DN.

It has been well established that the excessive production of inflammatory cytokines, reactive oxygen species (ROS) and lactate dehydrogenase (LDH) release can be induced under high glucose (HG) conditions, which may lead to podocyte depletion $(13,14)$. Emerging evidence has suggested that active components contained within certain Traditional Chinese Medicine exert suppressive effects on HG-induced inflammation and oxidative stress (14-16). Gastrodin is one of the primary components in the rhizome of the saprophytic perennial herb Gastrodia elata (17). A previous study demonstrated that gastrodin can exert anti-inflammatory and antioxidative effects on neurodegenerative diseases including Alzheimer's disease, Parkinson's disease and cerebral ischemia/reperfusion (18). Another study revealed that gastrodin treatment conferred neuroprotective effects on patients with type 2 diabetes by inhibiting endoplasmic reticulum stress and activating the NOD-, LRR- and pyrin domain-containing protein 3 (NLRP3) inflammasome (19). Additionally, gastrodin could 
suppress the apoptosis of human retinal endothelial cells by blocking the sirtuin $1 /$ Toll-like receptor $4 / \mathrm{NF}-\kappa \mathrm{B}$ p65 signaling pathway under HG conditions (20). However, to the best of our knowledge, the role of gastrodin in DN has not been previously investigated. Based on these aforementioned findings, the present study hypothesized that gastrodin possesses potential therapeutic effects against DN.

The AMP-activated protein kinase (AMPK)/nuclear factor erythroid 2-related factor 2 (Nrf2) signaling pathway has been reported to regulate several cellular processes, including inflammation, oxidative stress and apoptosis (21-23). A previous study has revealed that gastrodin ameliorated oxidative stress and the inflammatory response whilst improving lipid metabolism by activating the AMPK/Nrf2 pathway in nonalcoholic fatty liver disease mouse and rat models (24). Additionally, 4-O-methylhonokiol has been found to ameliorate type 2 diabetes-induced nephropathy in mice by activating AMPK-mediated fatty acid oxidation and Nrf2-mediated oxidative stress reduction (25). Therefore, it was speculated that gastrodin may inhibit podocyte inflammation, oxidative stress and apoptosis induced by HG through activation of the AMPK/Nrf2 pathway.

In the present study, immortalized mouse podocytes were pretreated with different doses of gastrodin under HG conditions to establish an in vitro model of podocyte injury. The aim was to reveal evidence to support the potential protective effects of gastrodin against inflammation and oxidative stress in podocytes induced by HG.

\section{Materials and methods}

Cell culture and treatment. The mouse podocyte MPC5 cell line was obtained from the American Type Culture Collection (26-28). Podocytes were maintained in RPMI-1640 medium (Thermo Fisher Scientific, Inc.) supplemented with $10 \%$ inactivated FBS (Thermo Fisher Scientific, Inc.) and $10 \mathrm{U} / \mathrm{ml}$ mouse recombinant interferon- $\gamma($ IFN- $\gamma$; Cell Signaling Technology, Inc.) at $33^{\circ} \mathrm{C}$ in a humidified atmosphere of $5 \% \mathrm{CO}_{2}$. When they reached $80 \%$ confluence, the podocytes were incubated in RPMI-1640 medium without IFN- $\gamma$ at $37^{\circ} \mathrm{C}$ for 10-14 days to induce podocyte differentiation (29).

MPC5 cells were then divided into the following groups: i) Normal glucose (NG; $5.5 \mathrm{mM}$ D-glucose); ii) mannitol (MA; $5.5 \mathrm{mM}$ glucose + $24.5 \mathrm{mM}$ D-MA; as osmotic control); iii) $\mathrm{HG}$ (30 mM D-glucose); and iv) $\mathrm{HG}+$ gastrodin (Sigma-Aldrich; Merck KGaA).

MPC5 cells were pretreated with different concentrations of gastrodin $(0.1,1,10$ and $100 \mu \mathrm{M})$ for $24 \mathrm{~h}$ prior to $\mathrm{HG}$ addition (30). MPC5 cells were also pretreated with $20 \mu \mathrm{M}$ Compound C (AMPK inhibitor, also known as dorsomorphin; Tocris Bioscience) for $1 \mathrm{~h}$ before $\mathrm{HG}$ treatment $(31,32)$ at $37^{\circ} \mathrm{C}$.

Cell Counting Kit-8 (CCK-8) assay. A CCK-8 assay kit (Beyotime Institute of Biotechnology) was used to evaluate cell viability, according to the manufacturer's protocols. Briefly, MPC5 cells (5x10 3 cells/well) were seeded into 96-well plates and treated with or without $\mathrm{HG}$ and gastrodin for $24 \mathrm{~h}$ at $37^{\circ} \mathrm{C}$. Subsequently, $10 \mu \mathrm{l} /$ well of CCK-8 solution was added into each well and the cells were incubated for an additional $2 \mathrm{~h}$ at $37^{\circ} \mathrm{C}$. The absorbance in each well was measured at a wavelength of $450 \mathrm{~nm}$ using a microplate reader (Thermo Fisher Scientific, Inc.).

ELISA. Following cell treatment, the secretion levels of TNF- $\alpha$, IL- $1 \beta$ and IL- 6 in the MPC5 cell culture supernatants were determined using corresponding ELISA kits (cat. nos. RAB0477, RAB0274 and RAB0308, respectively; Merck $\mathrm{KGaA}$ ), according to the manufacturer's protocols.

Western blot analysis. Following treatment, MPC5 cells were lysed with RIPA buffer (Beyotime Institute of Biotechnology) to extract total proteins. Protein concentration was determined using the BCA Protein Assay kit. After denaturation at $100^{\circ} \mathrm{C}$ for $10 \mathrm{~min}$, protein samples $(40 \mu \mathrm{g})$ from each group were loaded and separated using 10\% SDS-PAGE before they were transferred onto PVDF membranes. Following blocking with $5 \%$ skimmed milk for $2 \mathrm{~h}$ at room temperature, the membranes were incubated at $4^{\circ} \mathrm{C}$ overnight with primary antibodies (all from Abcam except cleaved caspase-6) against monocyte chemoattractant protein 1 (MCP-1; dilution, 1:1,000; cat. no. ab214819), NLRP3 (dilution, 1:1,000; cat. no. ab263899), apoptosis-associated speck-like protein (ASC; dilution, 1:1,000; cat. no. ab283684), caspase-1 (dilution, 1:1,000; cat. no. ab207802), Bcl-2 (dilution, 1:1,000; cat. no. ab32124), phosphorylated (p)-AMPK (dilution, 1:1,000; cat. no. ab92701), AMPK (dilution, 1:1,000; cat. no. ab110036), p-Nrf2 (dilution, 1:500; cat.no.ab76026),Nrf2 (dilution, 1:1,000; cat. no. ab137550), Bax (dilution, 1:1,000; cat. no. ab32503), cleaved caspase-3 (dilution, 1:500; cat. no. ab32042), caspase-3 (dilution, 1:1,000; cat. no. ab32351), cleaved caspase-6 (dilution, 1:500; cat. no. 9761; Cell Signaling Technology, Inc.), caspase-6 (dilution, 1:1,000; cat. no. ab108335), cleaved caspase-9 (dilution, 1:500; cat. no. ab2324), caspase-9 (dilution, 1:1,000; cat. no. ab32539) and heme oxygenase-1 (HO-1; dilution, 1:2,000; cat. no. ab52947). The membranes were then blotted with species-matched secondary antibodies (dilution, 1:1,000; cat. nos. ab6728 or ab6721; Abcam) for $1 \mathrm{~h}$ at room temperature. Protein bands were visualized using the ECL system (Pierce; Thermo Fisher. Scientific, Inc.), and the band density was analyzed using ImageJ software (version 1.49; National Institutes of Health).

LDH, malondialdehyde (MDA) and superoxide dismutase (SOD) level determination. The levels of LDH and MDA and the activity of SOD were evaluated using the corresponding assay kits according to the manufacturer's protocols. Briefly, MPC5 cells were seeded into six-well plates $\left(2 \times 10^{5}\right.$ cells/well $)$ and treated with or without $\mathrm{HG}$ and gastrodin for $24 \mathrm{~h}$ at $37^{\circ} \mathrm{C}$. Following treatment, cells were harvested and centrifuged at $4^{\circ} \mathrm{C}, 14,000 \mathrm{x}$ g for $5 \mathrm{~min}$ before the levels of $\mathrm{LDH}$ were measured in the cell supernatants using the LDH Assay kit (cat. no. C0016; Beyotime Institute of Biotechnology). Accordingly, the levels of MDA and SOD in the cell supernatants were determined using MDA and SOD Assay kits (cat. no. S0131S and S0086; both from Beyotime Institute of Biotechnology), respectively. A microplate reader was used to detect the absorbance in each well at a wavelength of $595 \mathrm{~nm}$.

TUNEL assay. Following treatment with HG and gastrodin, TUNEL staining assay was performed to evaluate the 
A

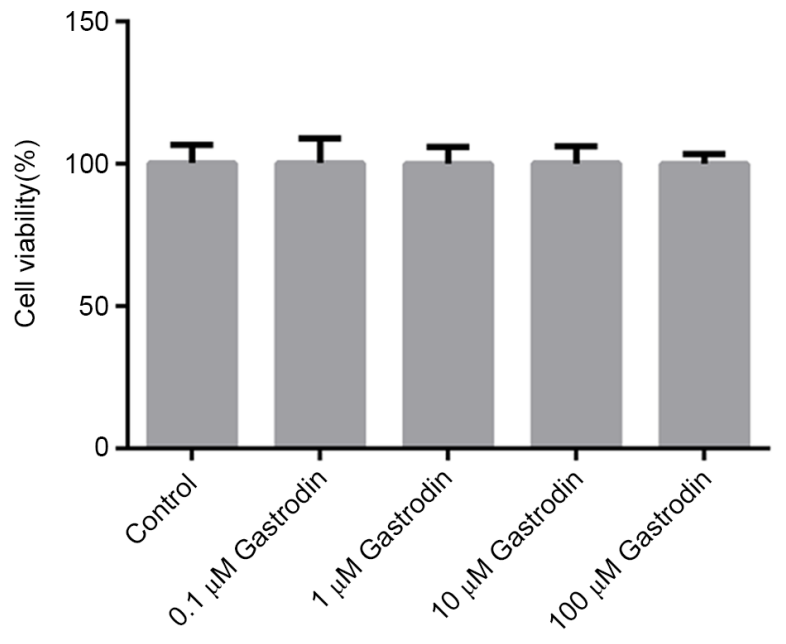

B

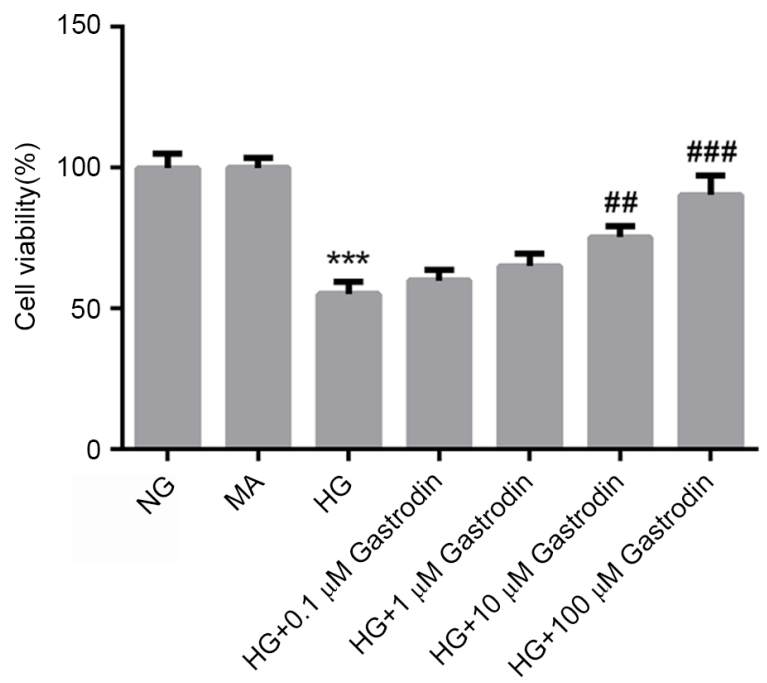

Figure 1. Gastrodin increases the viability of MPC5 cells under HG stimulation. The cell viability of cells (A) treated with increasing concentrations of gastrodin or (B) under HG with or without gastrodin pre-treatment was detected using Cell Counting Kit-8 assay. ${ }^{* * *} \mathrm{P}<0.001$ vs. NG. ${ }^{\# \#} \mathrm{P}<0.01$ and ${ }^{\# \# \#} \mathrm{P}<0.001$ vs. HG. NG, normal glucose; MA, mannitol; HG, high glucose.

apoptosis rate of MPC5 cells. The TUNEL Apoptosis Detection kit (cat. no. 11684817910; Roche Applied Science) was used according to the manufacturer's protocols. In brief, the cells were washed twice with PBS after the experimental procedures, then fixed with $4 \%$ paraformaldehyde at room temperature in the dark for $30 \mathrm{~min}$. Following incubation with proteinase $\mathrm{K}$ for $15 \mathrm{~min}$ at $37^{\circ} \mathrm{C}$, cells were placed in $3 \%$ $\mathrm{H}_{2} \mathrm{O}_{2}$ for $15 \mathrm{~min}$ at room temperature and stained using the TUNEL detection kit. Subsequently, cells were incubated with DAB for $10 \mathrm{~min}$, counterstained with hematoxylin for $2 \mathrm{~min}$ at room temperature and washed with PBS. Images from TUNEL-positive cells were captured under a light microscope (Olympus Corporation)at x200 magnification from five separate randomly selected fields. The TUNEL apoptosis rate $(\%)=$ number of TUNEL-positive podocytes/total number of podocytes $\mathrm{x} 100 \%$.

Statistical analysis. Data are presented as the mean \pm SEM from at least three independent experiments. All data were analyzed using the SPSS 19.0 software (IBM Corp.). Multiple group comparisons were analyzed by one-way ANOVA followed by Tukey's or Bonferroni post hoc tests. $\mathrm{P}<0.05$ was considered to indicate a statistically significant difference.

\section{Results}

Gastrodin increases the viability of $H G$-treated MPC5 cells. To evaluate the cytotoxicity of gastrodin, MPC5 cells were treated with different concentrations of gastrodin for $24 \mathrm{~h}$. CCK-8 assay results showed that treatment with gastrodin had no effect on cell viability (Fig. 1A). To examine the effects of gastrodin on cell viability under HG conditions, MPC5 cells were pre-treated with different doses of gastrodin prior to $\mathrm{HG}$ treatment. CCK-8 results showed that gastrodin treatment increased the viability of $\mathrm{HG}$-induced MPC5 cells in a dose-dependent manner, with the increases becoming significant at 10 and $100 \mu \mathrm{M}$ (Fig. 1B). Therefore, the dose of $100 \mu \mathrm{M}$ gastrodin was selected for subsequent experiments.

\section{Gastrodin alleviates $H G$-induced inflammation and oxidative} stress in MPC5 cells. To explore the effect of gastrodin in the development of DN, the concentration of inflammatory cytokines, oxidative stress status and the expression levels of NLRP3 signaling-related proteins were next determined. The ELISA results revealed that $\mathrm{HG}$ challenge significantly enhanced the secretion of TNF- $\alpha$, IL-1 $\beta$ and IL-6, whilst gastrodin treatment partially but significantly reversed this effect (Fig. 2A). Furthermore, western blot analysis results demonstrated that $\mathrm{HG}$ challenge significantly upregulated the protein expression levels of MCP-1, NLRP3, ASC and caspase-1, which may serve an important role in mediating the inflammatory responses (Fig. 2B). Pre-treatment with gastrodin also partially but significantly reversed the effects of HG on the expression of MCP-1, NLRP3, ASC and caspase-1. The activities of LDH and SOD, and the levels of MDA were subsequently detected as biomarkers of oxidative stress. As shown in Fig. 2C, HG challenge significantly enhanced MDA levels and LDH activity, but significantly suppressed SOD activity. These effects were partially but significantly reversed by gastrodin pre-treatment. These results suggested that gastrodin can alleviate HG-induced inflammation and oxidative stress in MPC5 cells.

Gastrodin inhibits HG-induced apoptosis in MPC5 cells. To investigate the effect of gastrodin further, cell apoptosis and the expression of apoptosis-related proteins were determined. TUNEL assay results showed that HG stimulation significantly promoted MPC5 cell apoptosis, whereas cell treatment with gastrodin significantly attenuated HG-induced apoptosis (Fig. 3A). Furthermore, the expression levels of the antiapoptotic protein Bcl-2 were significantly decreased in the HG group compared with those in the NG group. Additionally, the expression levels of the proapoptotic protein Bax and those of cleaved caspase- 3 , cleaved caspase- 6 and cleaved caspase- 9 

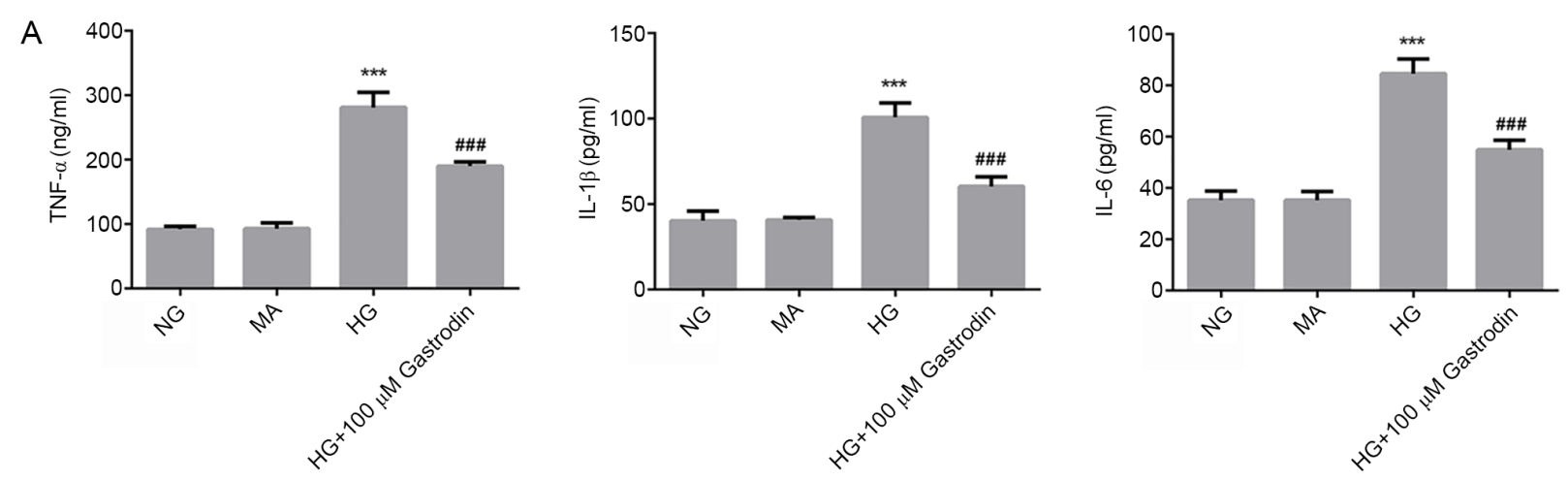

B
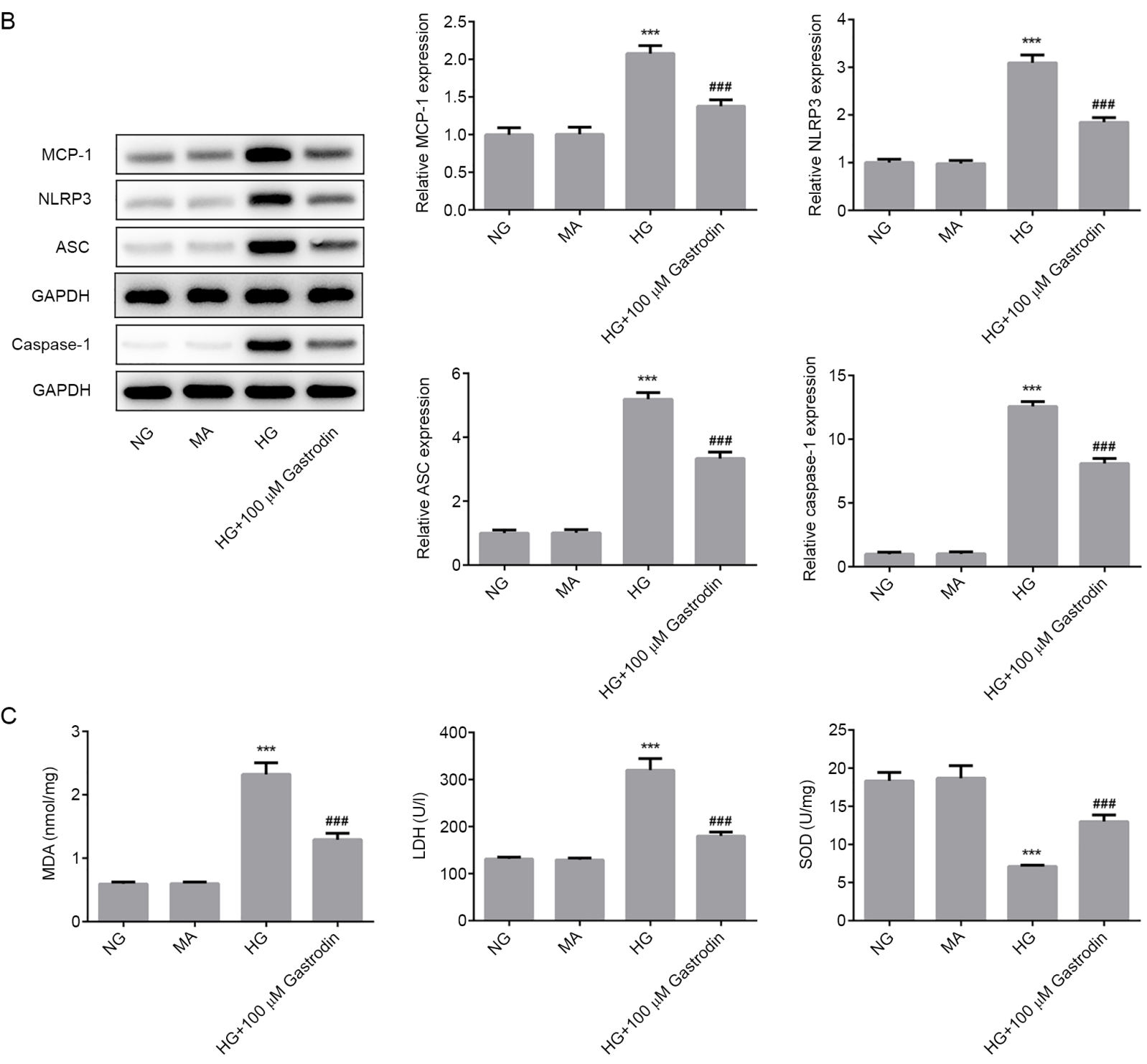

Figure 2. Gastrodin alleviates HG-induced inflammation and oxidative stress in MPC5 cells. (A) The secretion of inflammatory cytokines TNF- $\alpha$, IL-1 $\beta$ and IL-6 were analyzed by ELISA. (B) The expressions levels of MCP-1, NLRP3, ASC and Caspase-1 were determined by western blotting and quantified (C) MDA levels and the activities of LDH and SOD were quantified using their respective assay kits. ${ }^{* * *} \mathrm{P}<0.001$ vs. NG. ${ }^{\# \# \#} \mathrm{P}<0.001$ vs. HG. NG, normal glucose; HG, high glucose; MA, mannitol; LDH, lactate dehydrogenase; SOD, superoxide dismutase; MDA, malondialdehyde; MCP-1, monocyte chemoattractant protein 1; ASC, apoptosis-associated speck-like protein; NLRP3, NOD-, LRR- and pyrin domain-containing protein 3.

were all significantly increased in the HG group compared with those in the NG group. Gastrodin treatment significantly reversed the HG-induced Bcl-2 downregulation and upregulation of Bax, cleaved caspase- 3 , cleaved caspase- 6 and cleaved caspase-9 (Fig. 3B). These aforementioned findings suggest that gastrodin can inhibit HG-induced MPC5 cell apoptosis.
Gastrodin inhibits HG-induced inflammation, oxidative stress, and apoptosis by activating the AMPK/Nrf2 signaling pathway in MPC5 cells. To investigate the specific mechanism underlying the effect of gastrodin on podocyte injury, the protein levels of p-AMPK/AMPK, p-Nrf2/Nrf2 and HO-1 were detected by western blot analysis. HG challenge significantly reduced the 
A
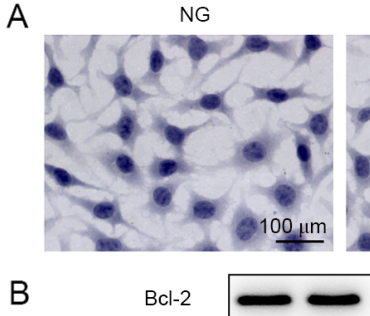

B

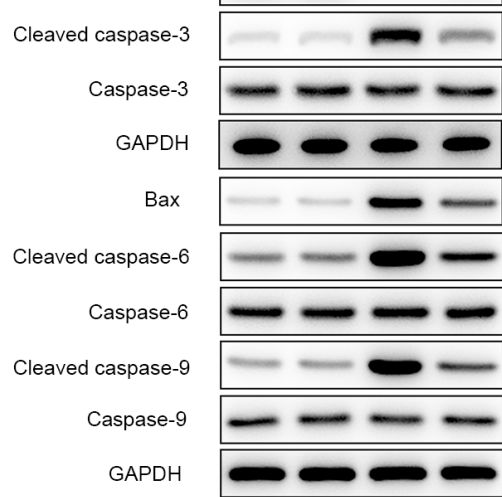

GAPDH

MA
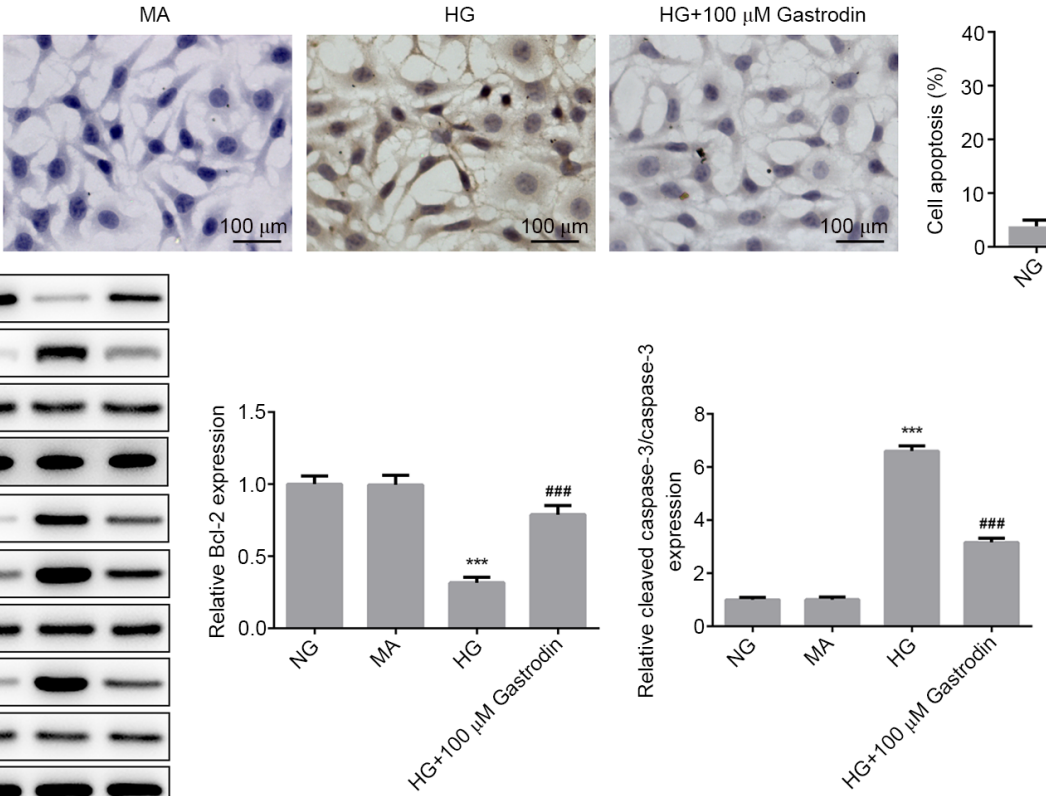

$H G+100 \mu M$ Gastrodin
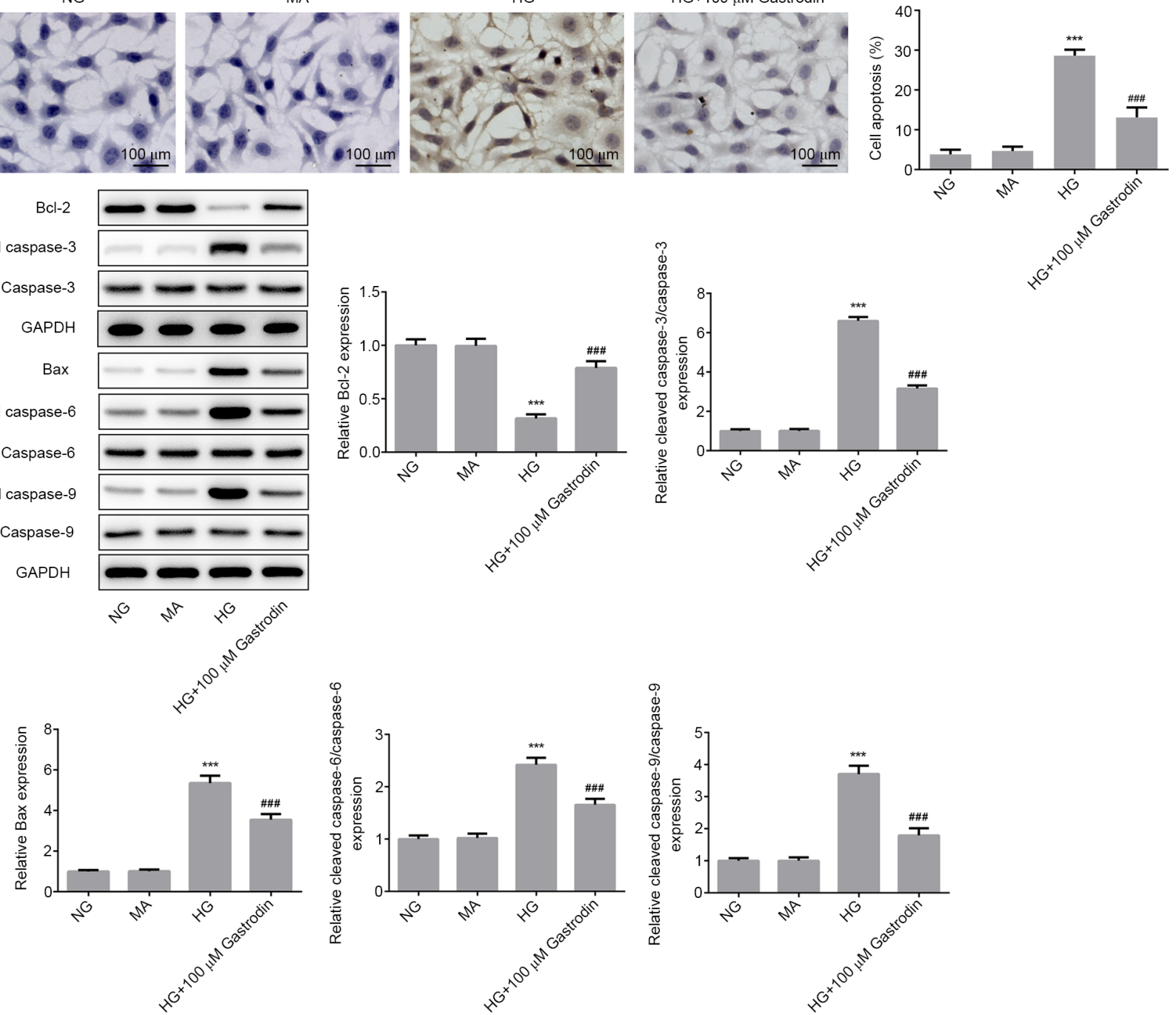

Figure 3. Gastrodin inhibits HG-induced cell apoptosis in MPC5 cells. (A) Cell apoptosis was measured by TUNEL assay. (B) The expressions levels of proteins related to apoptosis were determined by western blotting and quantified. ${ }^{* * *} \mathrm{P}<0.001 \mathrm{vs}$. NG. ${ }^{\# \#} \mathrm{P}<0.001 \mathrm{vs}$. HG. NG, normal glucose; HG, high glucose; MA, mannitol.

ratios of p-AMPK/AMPK and p-Nrf2/Nrf2 and the expression of HO-1 (Fig. 4). The levels of AMPK and Nrf2 phosphorylation and $\mathrm{HO}-1$ expression were found be significantly upregulated in the $\mathrm{HG}+100 \mu \mathrm{M}$ gastrodin group compared with those in the HG group (Fig. 4), suggesting that gastrodin can promote the activation of the AMPK/Nrf2 signaling pathway.

To verify the biochemical mechanism of gastrodin, cells were treated with compound $\mathrm{C}$ to inhibit AMPK. MPC5 cells were first pretreated with $20 \mu \mathrm{M}$ compound $\mathrm{C}$ for $1 \mathrm{~h}$ prior to HG treatment before the secretion levels of inflammatory cytokines, oxidative stress markers, cell apoptosis and the expression of apoptosis-related proteins were all measured. Pre-treatment with compound $\mathrm{C}$ significantly reversed the inhibitory effects of gastrodin on the secretion of inflammatory factors TNF- $\alpha$, IL-1 $\beta$ and IL-6 under HG (Fig. 5A). The expression levels of NLRP3, ASC and caspase-1 were significantly increased in the $\mathrm{HG}+100 \mu \mathrm{M}$ gastrodin + compound $\mathrm{C}$ group compared with those in the $\mathrm{HG}+100 \mu \mathrm{M}$ gastrodin group (Fig. 5B). Additionally, treatment with compound C significantly reversed the effects of gastrodin on MDA levels and activities of LDH and SOD (Fig. 5C).
A significantly increased apoptosis rate (Fig. 6A) and expression of proapoptotic proteins Bax, cleaved caspase-3, cleaved caspase- 6 and cleaved caspase-9 (Fig. 6B) were detected in cells treated with compound $\mathrm{C}$ compared with those in the $\mathrm{HG}+100 \mu \mathrm{M}$ gastrodin group. These findings suggest that gastrodin can attenuate HG-induced inflammation, oxidative stress and apoptosis through the activation of the AMPK/Nrf2 signaling pathway in MPC5 cells.

\section{Discussion}

DN is characterized by glomerular hypertrophy, decreased glomerular filtration, proteinuria and renal fibrosis and is recognized as a chronic complication of diabetes mellitus that can eventually lead to the loss of renal function (33). The diagnosis of DN is normally made based on microalbuminuria (34). Zhang et al (35) demonstrated that $20 \%$ of patients with diabetes may exhibit nephropathy complications and the prevalence of $\mathrm{DN}$ has been increasing rapidly in China (36). However, the pathogenic mechanism of DN remains poorly understood. Previous studies have shown 

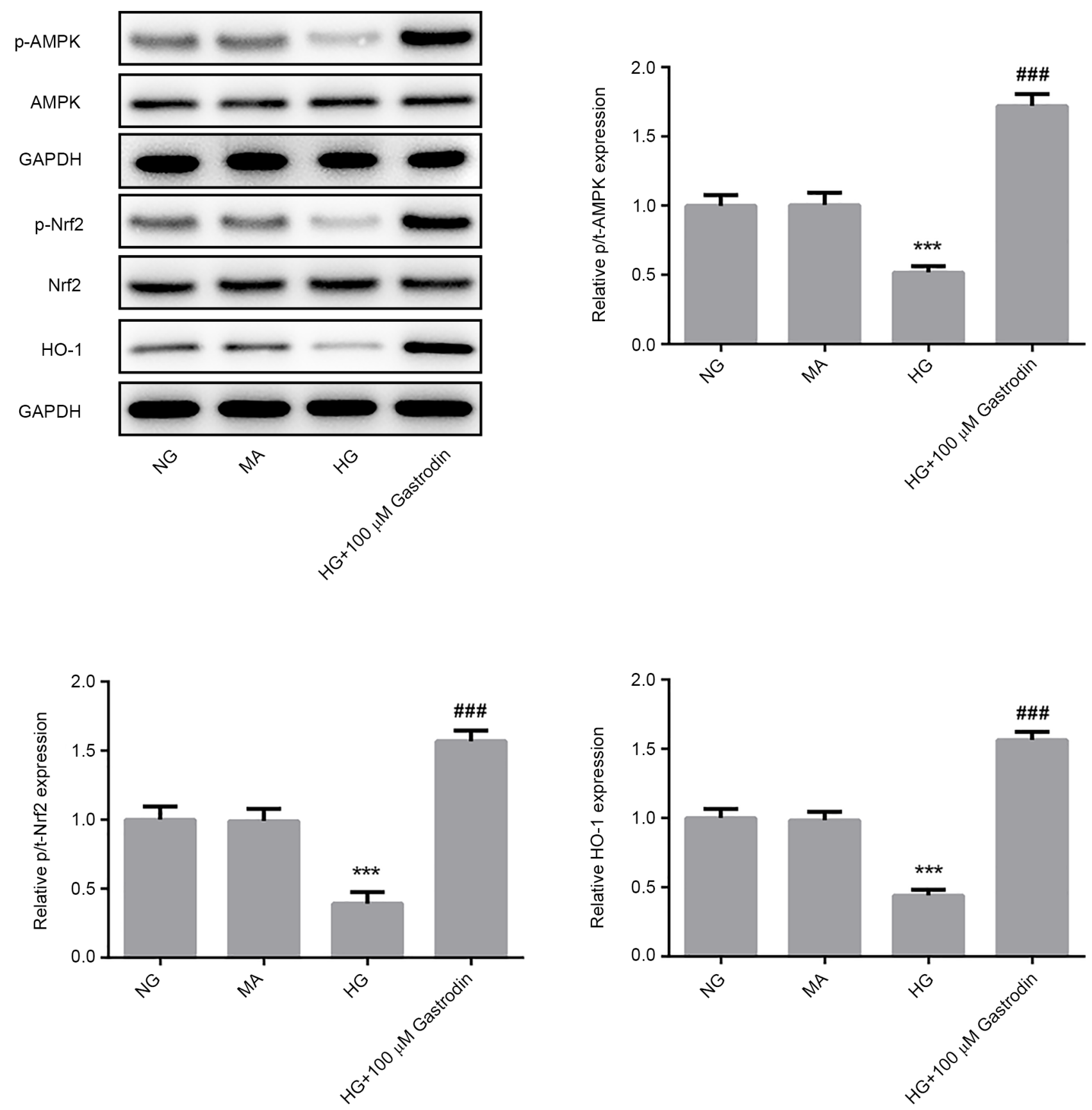

Figure 4. Gastrodin induces the activation of the AMPK/Nrf2 signaling pathway. The levels of p-AMPK/AMPK, p-Nrf2/Nrf2 and the expression of HO-1 were determined by western blotting and quantified. ${ }^{* * *} \mathrm{P}<0.001$ vs. NG and ${ }^{\# \# \#} \mathrm{P}<0.001$ vs. HG. NG, normal glucose; HG, high glucose; MA, mannitol; AMPK, 5'AMP-activated protein kinase; Nrf2, nuclear factor erythroid 2-related factor 2; HO-1, heme oxygenase-1; p-phosphorylated; t-, total.

that podocyte dysfunction serves a significant role in the development and progression of DN $(37,38)$. In addition, recent studies have suggested that gastrodin can exert a protective effect against HG-induced systemic organ injury $(19,20)$. Therefore, the present study aimed to explore the effect of gastrodin on podocyte injury and evaluate its potential mechanism. In the present study, 5.5 and $30 \mathrm{mM}$ glucose were used to simulate normal and high glucose conditions, respectively (39). The results revealed that gastrodin suppressed inflammation, oxidative stress and apoptosis in HG-stimulated podocytes by activating the AMPK/Nrf2 pathway, suggesting a protective effect of gastrodin on DN progression.
Emerging evidence has suggested that HG-induced oxidative stress is involved the development and progression of diabetes, where long-term oxidative stress under pathological conditions can induce excessive inflammatory responses (40). In addition, the multiprotein inflammasome complex, may serve an important role in initiating the inflammatory response. NLRP3 induces the recruitment and the autocatalytic activation of caspase-1, causing the formation of an inflammasome complex mediated by ASC (41). The formation of NLRP3 inflammasome and the activation of caspase-1 facilitates the processing of the cytosolic precursors of IL-1 $\beta$ and IL-18, resulting in the secretion of these biologically active cytokines (42). In the present study, HG 
A

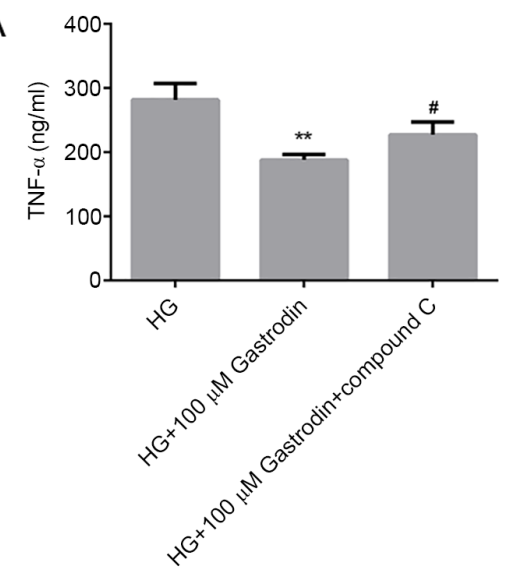

B

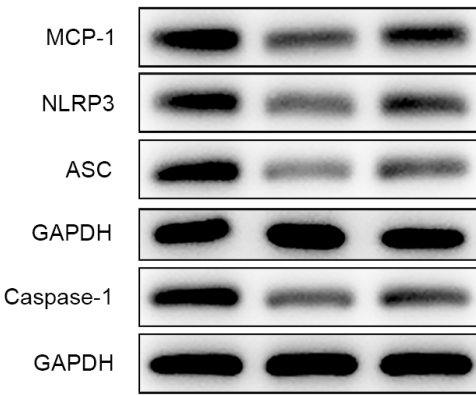

C

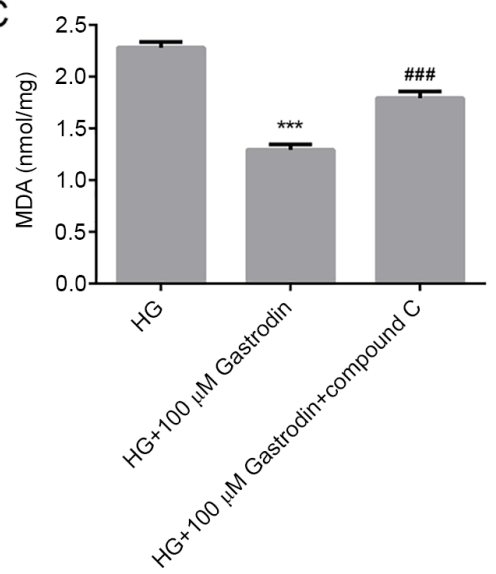

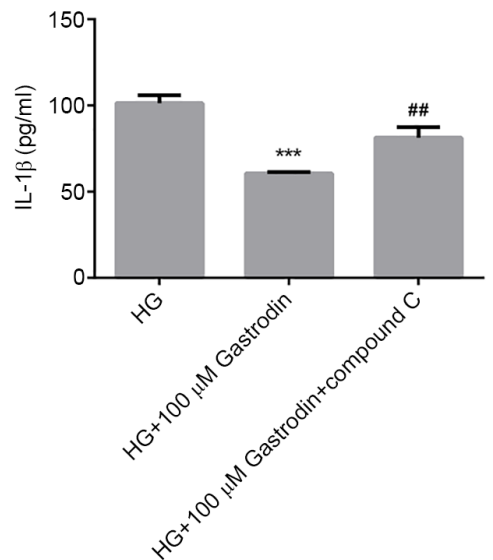
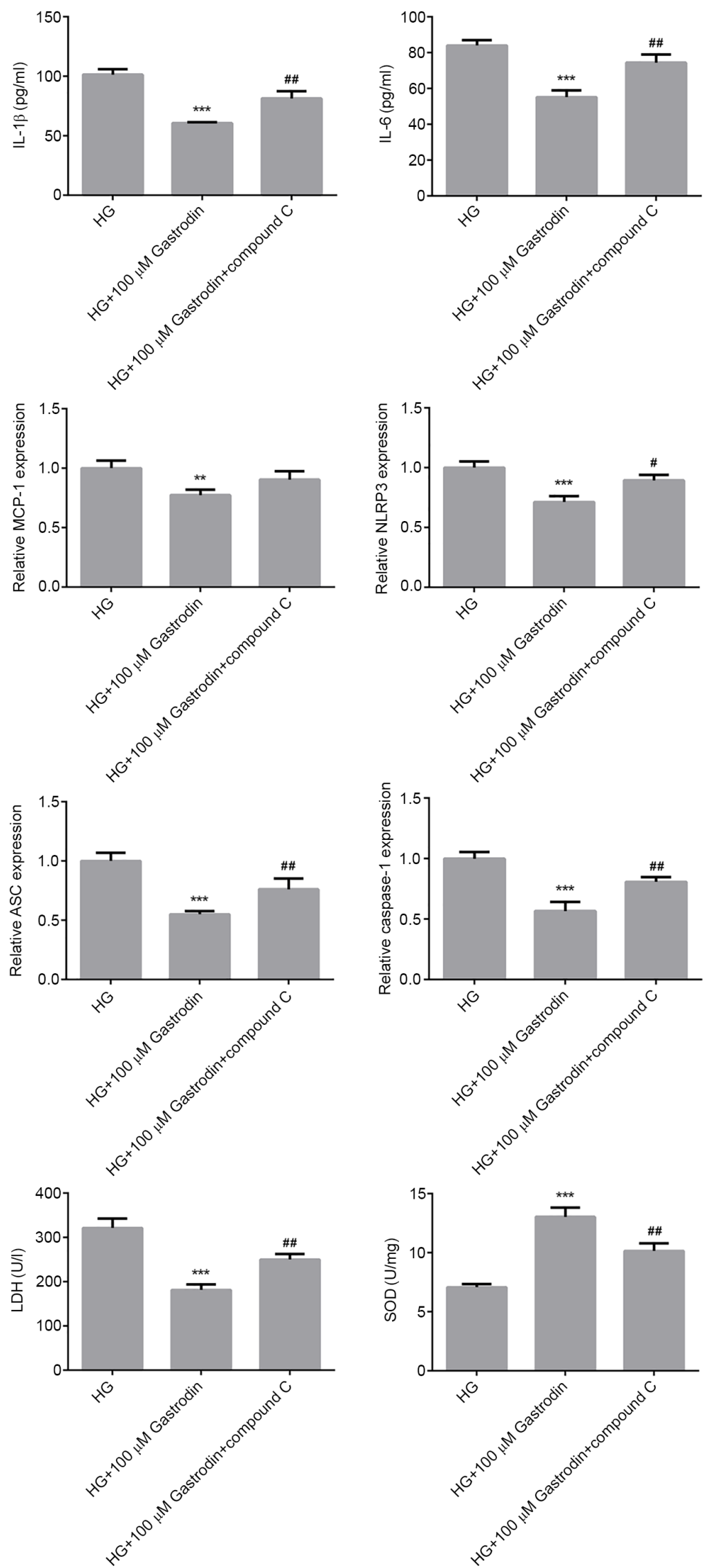

Figure 5. Gastrodin inhibits HG-induced inflammation and oxidative stress by activating the 5'AMP-activated protein kinase/nuclear factor erythroid 2-related factor 2 signaling pathway in MPC5 cells. (A) The secretion of inflammatory cytokines, TNF- $\alpha$, IL-1 $\beta$ and IL-6 were analyzed by ELISA. (B) The expression levels of MCP-1, NLRP3, ASC and Caspase-1 were determined by western blotting and quantified. (C) MDA levels and the activities of LDH and SOD were quantified using their respective assay kits. Error bars represent the mean \pm SEM from three independent experiments. ${ }^{* *} \mathrm{P}<0.01$ and ${ }^{* * *} \mathrm{P}<0.001 \mathrm{vs}$. HG. ${ }^{\#} \mathrm{P}<0.05$,

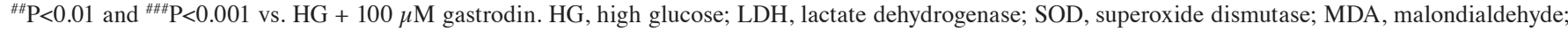
MCP-1, monocyte chemoattractant protein 1; ASC, apoptosis-associated speck-like protein; NLRP3, NOD-, LRR- and pyrin domain-containing protein 3. 
A

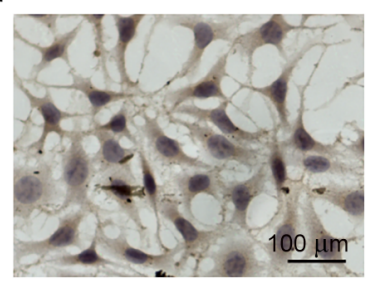

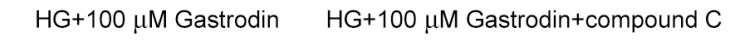
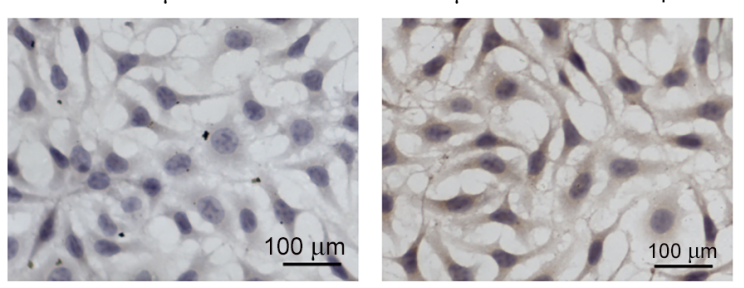

B
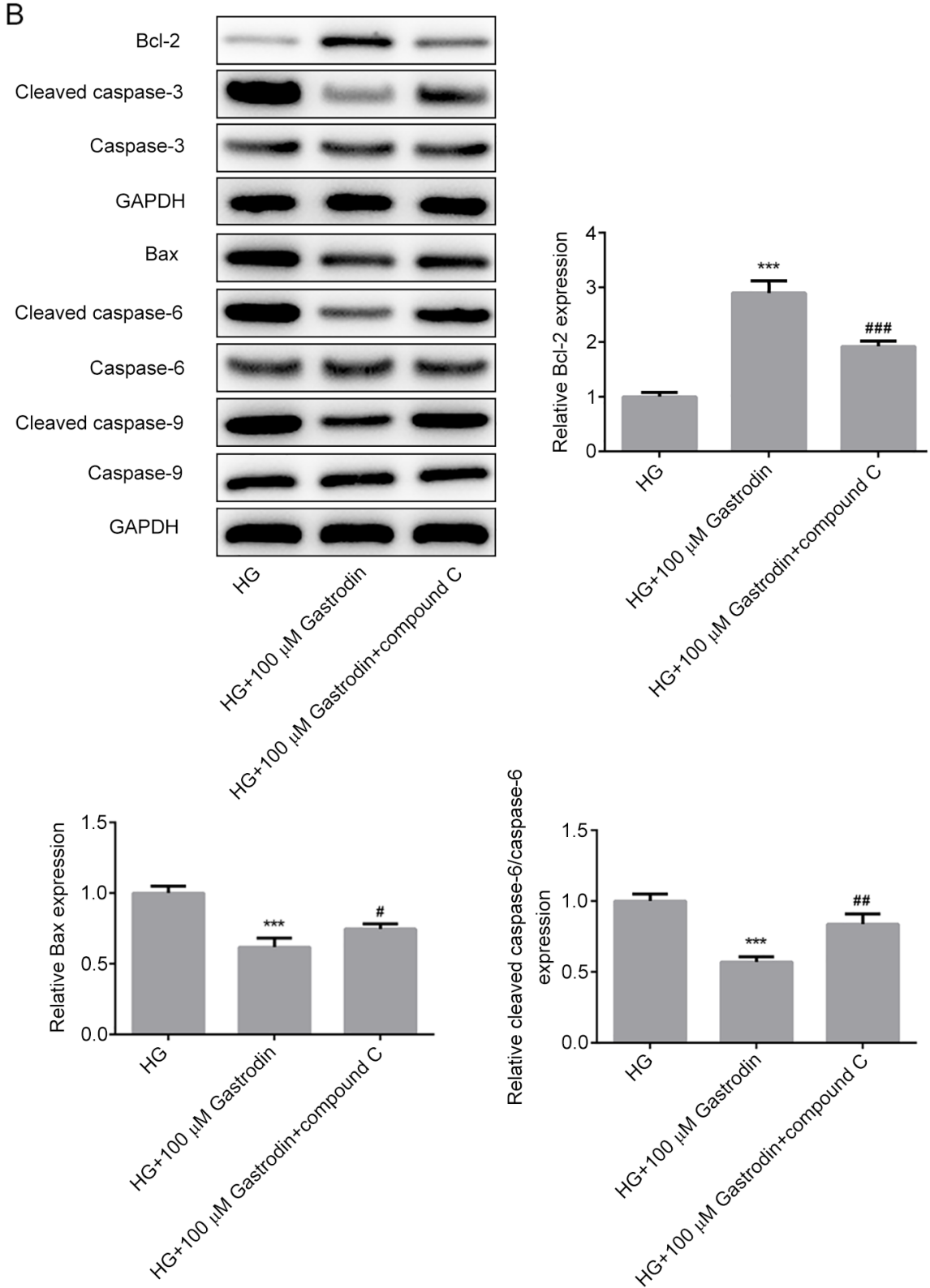
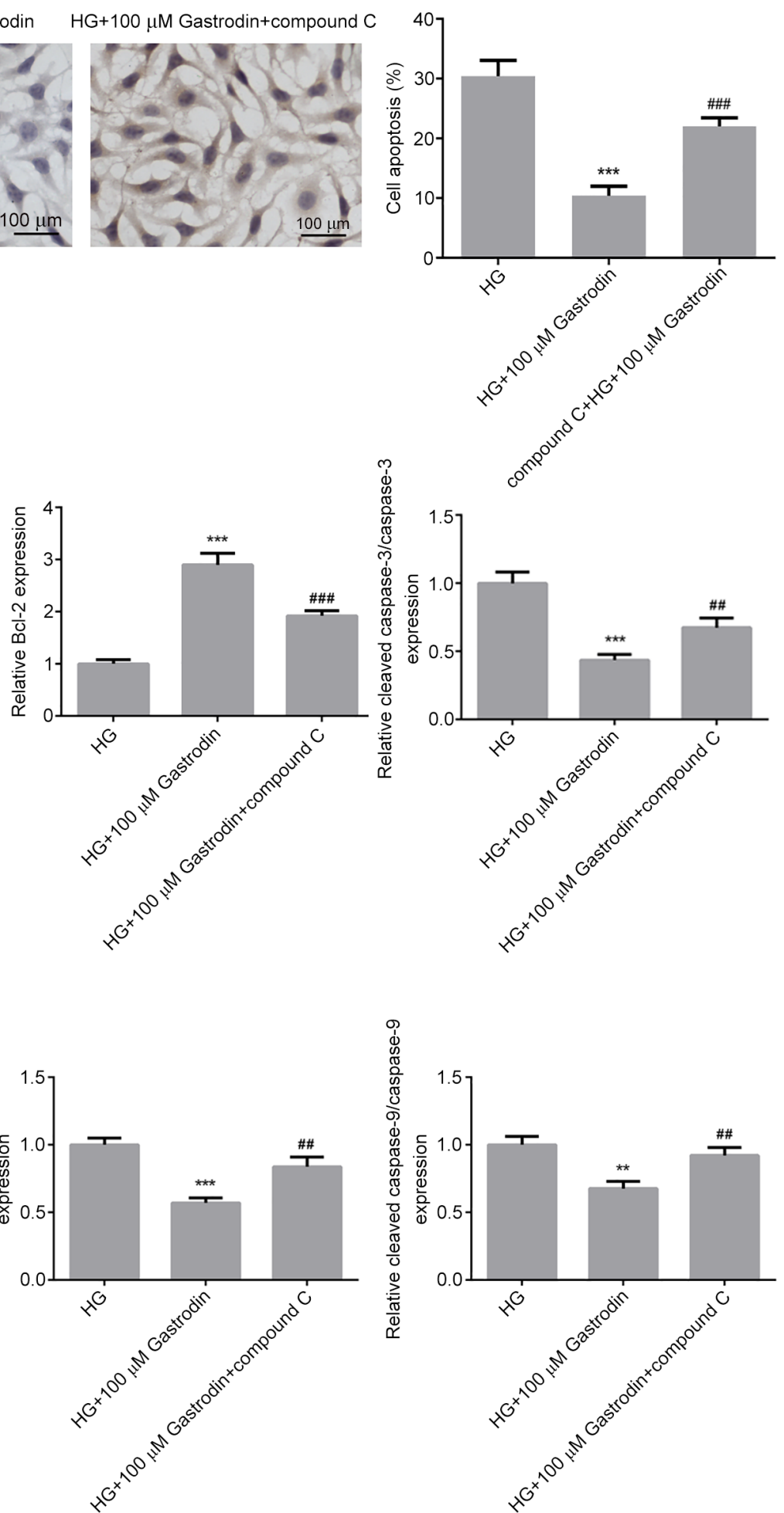

Figure 6. Gastrodin inhibits HG-induced apoptosis by activating the 5'AMP-activated protein kinase/nuclear factor erythroid 2-related factor 2 signaling pathway in MPC5 cells. (A) Cell apoptosis was analyzed using TUNEL assay. (B) The expressions levels of proteins related to apoptosis were determined by western blotting and quantified. ${ }^{* *} \mathrm{P}<0.01$ and ${ }^{* * * *} \mathrm{P}<0.001$ vs. HG. ${ }^{*} \mathrm{P}<0.05,{ }^{\# \#} \mathrm{P}<0.01$ and ${ }^{\# \# \#} \mathrm{P}<0.001$ vs. $\mathrm{HG}+100 \mu \mathrm{M}$ gastrodin. HG, high glucose.

challenge induced oxidative stress and promoted the secretion of inflammatory cytokines. In addition, gastrodin alleviated the HG-induced inflammation and oxidative stress in MPC5 cells, suggesting that gastrodin can exert therapeutic effects on DN. Inflammation and oxidative stress contribute to abnormal tubular cell cycle progression and disrupted cell proliferation, which have been proposed to be important mechanisms underlying multiple kidney diseases, including acute kidney injuries, chronic kidney diseases and polycystic kidney diseases (43). The results of the present study demonstrated that gastrodin dose-dependently increased the viability of HG-induced MPC5 cells. Furthermore, gastrodin inhibited HG-induced MPC5 cell apoptosis, suggesting that gastrodin can protect against HG-induced podocyte apoptosis. 
To investigate the specific mechanism underlying the protective effect of gastrodin on podocyte injury, the expression levels of the proteins associated with AMPK/Nrf2 signaling were determined. Accumulating evidence has suggested that the AMPK/Nrf2 signaling pathway serves a significant role in the pathogenesis of several inflammation-related diseases, such as atherosclerosis, diabetes and cancer (44-46). For example, gastrodin was found to reduce oxidative stress and inflammation, whilst improving lipid metabolism in a model of nonalcoholic fatty liver disease by activating AMPK/Nrf2 signaling (24). In the present study, gastrodin upregulated the phosphorylation levels of AMPK, Nrf2 and the expression of HO-1, which was consistent with the previous study aforementioned. Previous studies also reported that AMPK and Nrf2 can mediate fatty acid oxidation and reduce oxidative stress, respectively $(47,48)$. The activation of AMPK/Nrf2 signaling was previously found to improve lipid metabolism and attenuated oxidative stress in renal tissues of mice with type 2 diabetes-induced nephropathy $(25,49)$. Therefore, the present study hypothesized that gastrodin could inhibit the HG-triggered inflammation, oxidative stress and apoptosis by activating the AMPK/Nrf2 signaling pathway in MPC5 cells. To verify these hypotheses, MPC5 cells were treated with compound $\mathrm{C}$, an AMPK inhibitor. The results indicated that the inhibitory effects of gastrodin on HG-triggered inflammation, oxidative stress and apoptosis were overturned following cell exposure to compound $\mathrm{C}$. These findings suggested that gastrodin could protect against $\mathrm{HG}$-induced podocyte injury, inflammation and oxidative stress by activating the AMPK/Nrf2 signaling pathway, indicating this to a promising therapeutic approach for treating DN.

However, there are several limitations to the present study. The effects of gastrodin on DN were only observed in vitro. The role of gastrodin in animal DN models and human tissues with DN was not explored in the present study. CCK-8 assay was used to detect the effects of different doses of gastrodin on cell viability, which found that $0-100 \mu \mathrm{M}$ gastrodin had no significant effect on the cell viability of untreated MPC5 cells. Due to the substantial difference in the experiments in vitro and in vivo, the dose of gastrodin used in the present study can only serve as a reference for future in vitro experiments. The appropriate dose of gastrodin for clinical use in humans require further investigation. In addition, the focus of the present study was to investigate the effects of gastrodin on inflammation, oxidative stress and apoptosis of podocytes, but did not explore the effects of $\mathrm{HG}$ and gastrodin treatment on cell cycle progression. Finally, the expression of proteins in the AMPK/Nrf2 signaling pathway was mainly investigated in the present study, but it did not explore the effects of gastrodin on the Sirtuin-1 pathway, which is another signaling pathway that is associated with AMPK (50).

In summary, gastrodin treatment restored podocyte viability under $\mathrm{HG}$ conditions in a dose-dependent manner. Notably, gastrodin inhibited HG-induced inflammation, oxidative stress and podocyte apoptosis. Furthermore, gastrodin promoted the activation of the AMPK/Nrf2 signaling pathway in HG-stimulated podocytes. Collectively, these findings suggest that gastrodin can exhibit a protective effect against podocyte injury by activating AMPK/Nrf2 signaling in DN.
Therefore, gastrodin can be considered as an effective therapeutic agent for treating DN.

\section{Acknowledgements}

Not applicable.

\section{Funding}

No funding was received.

\section{Availability of data and materials}

The datasets used and/or analyzed during the current study are available from the corresponding author on reasonable request.

\section{Authors' contributions}

LH and YZ designed the experiments. LH and MS performed the experiments and analyzed the data. YZ and LH confirm the authenticity of all the raw data. All authors read and approved the final version of the manuscript.

\section{Ethics aproval and consent to participate}

Not applicable.

\section{Patient consent for publication}

Not applicable.

\section{Competing interests}

The authors declared that they have no competing interests.

\section{References}

1. Gnudi L, Coward RJM and Long DA: Diabetic nephropathy: Perspective on novel molecular mechanisms. Trends Endocrinol Metab 27: 820-830, 2016.

2. Yamahara K, Yasuda M, Kume S, Koya D, Maegawa H and Uzu T: The role of autophagy in the pathogenesis of diabetic nephropathy. J Diabetes Res 2013: 193757, 2013.

3. Gheith O, Farouk N, Nampoory N, Halim MA and Al-Otaibi T: Diabetic kidney disease: World wide difference of prevalence and risk factors. J Nephropharmacol 5: 49-56, 2016.

4. Feng Y, Weng H, Ling L, Zeng T, Zhang Y, Chen D and Li H: Modulating the gut microbiota and inflammation is involved in the effect of Bupleurum polysaccharides against diabetic nephropathy in mice. Int J Biol Macromol 132: 1001-1011, 2019.

5. Morigi M, Perico L, Corna D, Locatelli M, Cassis P, Carminati CE, Bolognini S, Zoja C, Remuzzi G, Benigni A and Buelli S: C3a receptor blockade protects podocytes from injury in diabetic nephropathy. JCI Insight 5: e131849, 2020.

6. Zhang P, Fang J, Zhang J, Ding S and Gan D: Curcumin inhibited podocyte cell apoptosis and accelerated cell autophagy in diabetic nephropathy via regulating Beclin1/UVRAG/Bcl2 . Diabetes Metab Syndr Obes 13: 641-652, 2020

7. Kim YI, Kim CH, Choi CS, Chung YE, Lee MS, Lee SI, Park JY, Hong SK and Lee KU: Microalbuminuria is associated with the insulin resistance syndrome independent of hypertension and type 2 diabetes in the Korean population. Diabetes Res Clin Pract 52: 145-152, 2001.

8. Al-Rubeaan K, Siddiqui K, Alghonaim M, Youssef AM and AlNaqeb D: The Saudi diabetic kidney disease study (Saudi-DKD): Clinical characteristics and biochemical parameters. Ann Saudi Med 38: 46-56, 2018. 
9. Lopes TG, de Souza ML, da Silva VD, Dos Santos M, da Silva WIC, Itaquy TP, Garbin HI and Veronese FV: Markers of renal fibrosis: How do they correlate with podocyte damage in glomerular diseases? PLoS One 14: e0217585, 2019.

10. Chen Y, Lin L, Tao X, Song Y, Cui J and Wan J: The role of podocyte damage in the etiology of ischemia-reperfusion acute kidney injury and post-injury fibrosis. BMC Nephrol 20: 106, 2019.

11. Ilatovskaya DV, Blass G, Palygin O, Levchenko V, Pavlov TS, Grzybowski MN, Winsor K, Shuyskiy LS, Geurts AM, Cowley AW Jr, et al: A NOX4/TRPC6 pathway in podocyte calcium regulation and renal damage in diabetic kidney disease. J Am Soc Nephrol 29: 1917-1927, 2018.

12. Khalilpourfarshbafi M, Hajiaghaalipour F, Selvarajan KK and Adam A: Mesenchymal stem cell-based therapies against podocyte damage in diabetic nephropathy. Tissue Eng Regen Med 14: 201-210, 2017.

13. Chen Y, Liu Q, Shan Z, Zhao Y, Li M, Wang B, Zheng X and Feng W: The protective effect and mechanism of catalpol on high glucose-induced podocyte injury. BMC Complement Altern Med 19: 244, 2019.

14. Zhan X, Yan C, Chen Y, Wei X, Xiao J, Deng L, Yang Y, Qiu P and Chen Q: Celastrol antagonizes high glucose-evoked podocyte injury, inflammation and insulin resistance by restoring the HO-1-mediated autophagy pathway. Mol Immunol 104: 61-68, 2018.

15. Yu Q, Zhang M, Qian L, Wen D and Wu G: Luteolin attenuates high glucose-induced podocyte injury via suppressing NLRP3 inflammasome pathway. Life Sci 225: 1-7, 2019.

16. Zhong Y, Lee K, Deng Y, Ma Y, Chen Y, Li X, Wei C, Yang S, Wang T, Wong NJ, et al: Arctigenin attenuates diabetic kidney disease through the activation of PP2A in podocytes. Nat Commun 10: 4523, 2019.

17. Xu CB, Guo QL, Wang YN, Lin S, Zhu CG and Shi JG: Gastrodin derivatives from gastrodia elata. Nat Prod Bioprospect 9: 393-404, 2019

18. Liu Y, Gao J, Peng M, Meng H, Ma H, Cai P, Xu Y, Zhao Q and Si G: A review on central nervous system effects of gastrodin. Front Pharmacol 9: 24, 2018.

19. Ye T, Meng X, Zhai Y, Xie W, Wang R, Sun G and Sun X Gastrodin ameliorates cognitive dysfunction in diabetes rat model via the suppression of endoplasmic reticulum stress and NLRP3 inflammasome activation. Front Pharmacol 9: 1346, 2018.

20. Zhang TH, Huang CM, Gao X, Wang JW, Hao LL and Ji Q: Gastrodin inhibits high glucose-induced human retinal endothelial cell apoptosis by regulating the SIRT1/TLR4/NF- $\mathrm{kBp} 65$ signaling pathway. Mol Med Rep 17: 7774-7780, 2018.

21. Cheng Y, Qi Y,Liu S, Di R, Shi Q, Li J and Pei C: Clq/TNF-related protein 9 inhibits high glucose-induced oxidative stress and apoptosis in retinal pigment epithelial cells through the activation of AMPK/Nrf2 signaling pathway. Cell Transplant 29: 963689720962052,2020

22. $\mathrm{Xu} \mathrm{W}$, Zhao $\mathrm{T}$ and $\mathrm{Xiao} \mathrm{H}$ : The implication of oxidative stress and AMPK-Nrf2 antioxidative signaling in pneumonia pathogenesis. Front Endocrinol (Lausanne) 11: 400, 2020.

23. Zhou F, Wang M, Ju J, Wang Y, Liu Z, Zhao X, Yan Y, Yan S, Luo X and Fang Y: Schizandrin A protects against cerebral ischemia-reperfusion injury by suppressing inflammation and oxidative stress and regulating the AMPK/Nrf2 pathway regulation. Am J Transl Res 11: 199-209, 2019.

24. Qu LL, Yu B, Li Z, Jiang WX, Jiang JD and Kong WJ: Gastrodin ameliorates oxidative stress and proinflammatory response in nonalcoholic fatty liver disease through the AMPK/Nrf2 pathway. Phytother Res 30: 402-411, 2016.

25. Ma T, Zheng Z, Guo H, Lian X, Rane MJ, Cai L, Kim KS, Kim KT, Zhang Z and Bi L: 4-O-methylhonokiol ameliorates type 2 diabetes-induced nephropathy in mice likely by activation of AMPK-mediated fatty acid oxidation and Nrf2-mediated anti-oxidative stress. Toxicol Appl Pharmacol 370: 93-105, 2019.

26. Wang RM, Wang ZB, Wang Y, Liu WY, Li Y, Tong LC, Zhang S, Su DF, Cao YB, Li L and Zhang LC: Swiprosin-1 promotes mitochondria-dependent apoptosis of glomerular podocytes via P38 MAPK pathway in early-stage diabetic nephropathy. Cell Physiol Biochem 45: 899-916, 2018

27. Han X, Li Q, Wang C and Li Y: MicroRNA-204-3p attenuates high glucose-induced MPC5 podocytes apoptosis by targeting braykinin B2 receptor. Exp Clin Endocrinol Diabetes 127: 387-395, 2019.

28. Tu Q, Li Y, Jin J, Jiang X, Ren Y and He Q: Curcumin alleviates diabetic nephropathy via inhibiting podocyte mesenchymal transdifferentiation and inducing autophagy in rats and MPC5 cells. Pharm Biol 57: 778-786, 2019.
29. Cao Y, Hao Y, Li H, Liu Q, Gao F, Liu W and Duan H: Role of endoplasmic reticulum stress in apoptosis of differentiated mouse podocytes induced by high glucose. Int J Mol Med 33: 809-816, 2014

30. Xi Z, Qiao Y, Wang J, Su H, Bao Z, Li H, Liao X and Zhong X: Gastrodin relieves inflammation injury induced by lipopolysaccharides in MRC-5 cells by up-regulation of miR-103. J Cell Mol Med 24: 1451-1459, 2020

31. Kim SH, Hwang JT, Park HS, Kwon DY and Kim MS: Capsaicin stimulates glucose uptake in $\mathrm{C} 2 \mathrm{C} 12$ muscle cells via the reactive oxygen species (ROS)/AMPK/p38 MAPK pathway. Biochem Biophys Res Commun 439: 66-70, 2013.

32. Chuang KC, Chen FW, Tsai MH and Shieh JJ: EGR-1 plays a protective role in AMPK inhibitor compound C-induced apoptosis through ROS-induced ERK activation in skin cancer cells. Oncol Lett 21: 304, 2021.

33. Bhattacharjee N, Barma S, Konwar N, Dewanjee S and Manna P. Mechanistic insight of diabetic nephropathy and its pharmacotherapeutic targets: An update. Eur J Pharmacol 791: 8-24, 2016.

34. Papadopoulou-Marketou N, Chrousos GP and Kanaka-Gantenbein C: Diabetic nephropathy in type 1 diabetes: A review of early natural history, pathogenesis, and diagnosis. Diabetes Metab Res Rev 33: e2841, 2017.

35. Zhang XX, Kong J and Yun K: Prevalence of diabetic nephropathy among patients with type 2 diabetes mellitus in China: A meta-analysis of observational studies. J Diabetes Res 2020: 2315607, 2020.

36. Xiong $\mathrm{Y}$ and Zhou L: The signaling of cellular senescence in diabetic nephropathy. Oxid Med Cell Longev 2019: 7495629, 2019.

37. Bose M, Almas S and Prabhakar S: Wnt signaling and podocyte dysfunction in diabetic nephropathy. J Investig Med 65 1093-1101, 2017

38. Tung CW, Hsu YC, Shih YH, Chang PJ and Lin CL: Glomerular mesangial cell and podocyte injuries in diabetic nephropathy. Nephrology (Carlton) 23 (Suppl 4): S32-S37, 2018.

39. Wang S, Zhao X, Yang S, Chen B and Shi J: Salidroside alleviates high glucose-induced oxidative stress and extracellular matrix accumulation in rat glomerular mesangial cells by the TXNIP-NLRP3 inflammasome pathway. Chem Biol Interact 278: 48-53, 2017.

40. Zhao MX, Zhou B, Ling L, Xiong XQ, Zhang F, Chen Q, Li YH, Kang YM and Zhu GQ: Salusin- $\beta$ contributes to oxidative stress and inflammation in diabetic cardiomyopathy. Cell Death Dis 8: e2690, 2017.

41. Sutterwala FS, Ogura Y, Szczepanik M, Lara-Tejero M, Lichtenberger GS, Grant EP, Bertin J, Coyle AJ, Galán JE, Askenase PW and Flavell RA: Critical role for NALP3/CIAS1/Cryopyrin in innate and adaptive immunity through its regulation of caspase-1. Immunity 24: 317-327, 2006.

42. Pétrilli V, Dostert C, Muruve DA and Tschopp J: The inflammasome: A danger sensing complex triggering innate immunity Curr Opin Immunol 19: 615-622, 2007.

43. Lin TA, Wu VC and Wang CY: Autophagy in chronic kidney diseases. Cells 8: 61, 2019.

44. Kosuru R, Kandula V, Rai U, Prakash S, Xia Z and Singh S: Pterostilbene decreases cardiac oxidative stress and inflammation via activation of $\mathrm{AMPK} / \mathrm{Nrf} 2 / \mathrm{HO}-1$ pathway in fructose-fed diabetic rats. Cardiovasc Drugs Ther 32: 147-163, 2018.

45. Tanaka M, Kishimoto Y, Sasaki M, Sato A, Kamiya T, Kondo K and Iida K: Terminalia bellirica (Gaertn.) Roxb. extract and gallic acid attenuate LPS-induced inflammation and oxidative stress via MAPK/NF- $\kappa$ B and Akt/AMPK/Nrf2 pathways. Oxid Med Cell Longev 2018: 9364364, 2018.

46. Wang Y, Huang Y, Xu Y, Ruan W, Wang H, Zhang Y, Saavedra JM, Zhang L, Huang Z and Pang T: A Dual AMPK/Nrf2 activator reduces brain inflammation after stroke by enhancing microglia M2 polarization. Antioxid Redox Signal 28: 141-163, 2018.

47. Zeng HL, Huang SL, Xie FC, Zeng LM, Hu YH and Leng Y: Yhhu981, a novel compound, stimulates fatty acid oxidation via the activation of AMPK and ameliorates lipid metabolism disorder in ob/ob mice. Acta Pharmacol Sin 36: 343-352, 2015.

48. Zhan X, Li J and Zhou T: Targeting Nrf2-mediated oxidative stress response signaling pathways as new therapeutic strategy for pituitary adenomas. Front Pharmacol 12: 565748, 2021.

49. Kim Y, Lee H, Kim SY and Lim Y: Effects of lespedeza bicolor extract on regulation of AMPK associated hepatic lipid metabolism in type 2 diabetic mice. Antioxidants (Basel) 8: 599, 2019.

50. Huang Q, Wang T, Yang L and Wang HY: Ginsenoside Rb2 alleviates hepatic lipid accumulation by restoring autophagy via induction of Sirtl and activation of AMPK. Int J Mol Sci 18: 1063, 2017.

This work is licensed under a Creative Commons Attribution-NonCommercial-NoDerivatives 4.0 International (CC BY-NC-ND 4.0) License. 